\title{
An Efficient Screening Approach to be Used in Plasma Modeling and Ion-Surface Collision Experiments
}

\author{
J. Pomarico, D. I. Iriarte, and H. O. Di Rocco \\ Instituto de Física Arroyo Seco \\ Facultad de Ciencias Exactas, UNCPBA \\ Pinto 399, (7000) Tandil, Buenos Aires, Argentina
}

Received on 22 November, 2003

\begin{abstract}
In this work we show that the Layzer theory for atomic calculations provides a theoretical framework and also a powerful computational approach if correct rules for the calculation of the screening parameters are given. Using the virial as a model for potential energy and splitting of two-body operators as sum of onebody operators, a neat definition of screening is given, satisfying diverse physically indispensable properties. Many different experimental and theoretical results are reproduced with high accuracy, with no fitting procedure involving energy levels or numerical potentials. A $C++$ code and an executable file are available upon request.
\end{abstract}

\section{Introduction}

The use of screened hydrogenic models (SHM) for modeling plasmas and surface ion collision experiments has been well documented in recent works [1], [2]. Different methods are available for systematic numerical calculations of atomic parameters, based on the self-consistent Hartree-Fock procedure [3] [4]. However, it is important to have an efficient analytical approach, suitable for the calculation of energies of a large number of excited states. The purpose of this article is to give explicit formulas for the average energies of arbitrary complex configurations, specially of medium to highly ionized atoms. However, also good results for neutral and almost neutral atoms are obtained.

The starting point is the Layzer's formulation of the Zdependent theory of the many-electron atom truncated to second order [4] [5] [6]. Many authors pointed out in the past that the screening approximation is not very useful; however, that statement is not right provided that correct rules are given for calculating the screening. Moreover, relativistic corrections to the energy can be easily made and configuration interaction effects can be properly considered. It is important to point out that several published screening rules produce negative ionization potentials (IP) for neutral or few ionized systems [2] [7]. Other authors obtain the screening parameters either in order to reproduce the IP's calculated by Hartree-Fock [9] [10] or by adjusting analytical potentials with numerical results [11].

In this work, no empirical adjustments are made nor negative IP's emerge from our calculations. For the screening parameters, we use the approach due to Kregar [12] generalized by one of us [13], based on the splitting of the twobody potential energy operators into the sum of effective one-body operators. Total binding energies, ionization potentials and energies of complex excited configurations are in good or very good agreement with both, theoretical and experimental values.

In the following, we use the atomic units, where the electron charge and mass and the Dirac constant are equal to one ( $e=m_{e}=\hbar=1$ ) and the energies are measured in Hartrees: $1 \mathrm{Ht} \equiv 27.21 \mathrm{eV}$. A numerical code written in $C++$ and an executable file are available upon request.

\section{A Brief Summary of the Theory}

\subsection{The $Z^{-1}$ expansion}

Layzer's formulation of the Z-dependent theory of the many-electron atom is the starting point of our work [4], [5] [6]. The approximate non-relativistic Hamiltonian in atomic units given by

$$
H(N, Z)=-\frac{1}{2} \sum_{i=1}^{N}\left(\nabla_{i}^{2}+\frac{2 Z}{r_{i}}\right)+\sum_{i>j} \frac{1}{r_{i j}} .
$$

Introducing the new variable $\rho=Z r$, it can be shown that the total non-relativistic energy can be written as the expansion [4]

$$
E(N, Z)=Z^{2} E_{0}+Z E_{1}-E_{2}+Z^{-1} E_{3} \ldots
$$

where, exactly

$$
E_{0}=\left\langle\psi_{0}\left|H_{0}\right| \psi_{0}\right\rangle=-\frac{1}{2} \sum_{i=1}^{N} \frac{1}{n_{i}^{2}}
$$

and

$$
E_{1}=\left\langle\psi_{0}\left|H_{1}\right| \psi_{0}\right\rangle
$$


$E_{1}$ is given by the sum of the average Coulomb energy for electron pairs $\{i j\}$ (see below, Equation 10), and therefore in terms of the Slater's integrals $F^{k}$ and $G^{k}$, evaluated with hydrogenic wavefunctions with $Z=1$ [3][14]. For nonequivalent orbitals:

$$
\{i j\}=F^{0}(i j)-\sum_{k} g_{k} G^{k}(i j)
$$

whereas for the equivalent ones:

$$
\{i i\}=F^{0}(i i)-\sum_{k>0} f_{k} F^{k}(i i)
$$

the coefficients $g_{k}$ and $f_{k}$ can be found in the above cited references.

If we restrict the expansion (2) up to $E_{2}$, the energy can be written as

$$
E=-\sum_{n, l} \frac{w_{n l}\left(Z-\sigma_{n l}\right)^{2}}{2 n^{2}}
$$

where $w_{n l}$ is the number of electrons in the $n, l$ shell and $\sigma_{n l}$ is the corresponding screening parameter. Comparing Equations (2) and (7), we find that the $\sigma_{n l}$ 's satisfy

$$
E_{1}=\sum_{n, l} \frac{w_{n l}}{n^{2}} \sigma_{n l}
$$

and

$$
E_{2}=\sum_{n, l} \frac{w_{n l}}{2 n^{2}} \sigma_{n l}^{2}
$$

Then, to second-order approximation in the non-relativistic context, the average energy of a configuration is given by

$$
\begin{aligned}
E_{a v}= & -\frac{Z^{2}}{2}\left\{w_{1 s}+\frac{w_{2 s}+w_{2 p}}{4}+\frac{w_{3 s}+w_{3 p}+w_{3 d}}{9}+\ldots\right\} \\
& +Z\left\{\frac{1}{2} \sum_{i} w_{i}\left(w_{i}-1\right)\{i i\}+\sum_{i, j} w_{i} w_{j}\{i j\}\right\}-E_{2}
\end{aligned}
$$

where $w_{i}$ is a short notation for $w_{n_{i} l_{i}}$, the number of electrons in the $n_{i}, l_{i}$ shell.

Comparing Equation 8 with Equation 10, we see that $E_{1}$ can be calculated by two ways: in terms of the Slater integrals $F^{k}$ and $G^{k}$ or in terms of the $\sigma$ 's. Indeed, if we are capable of select a good method for $\sigma^{\prime} s$ calculations, the two values must be nearly equal. One of the merits of this work is the algorithm used for this calculus.

\subsection{The calculation and desirable properties of the screening parameters}

The concept of screening (and screened orbitals) is of old data and it is impossible to give a short account in this paper. In the past, screening parameters were obtained using spectroscopic data, numerical calculations and theoretical approaches. A short review can be found in the paper from one of the authors [6].

Screening parameters must have several indispensable properties: i) it must be derived theoretically in a neat form, ii) it must be dependent on $Z$ and $N$, accounting for the contraction of the orbitals, iii) the sum given by Equation 8 must be very nearly to the value $\frac{1}{2} \sum_{i} w_{i}\left(w_{i}-1\right)\{i i\}+$ $\sum_{i, j} w_{i} w_{j}\{i j\}$; this property is verified and it is exact for $Z \stackrel{i, j}{\rightarrow} \infty$, when $\sigma \rightarrow \sigma_{0}$ (see below, Eq. 11), iv) the corresponding values of $E_{2}$ must follow a law of the type $E_{2} \sim a+b / Z$ in order to reproduce the non-relativistic calculations using the Cowan 's code (see below, section Results), v) it must satisfy the $Z$ expansion

$$
\sigma(Z)=\sigma_{0}+\sigma_{1} Z^{-1}+\ldots
$$

We use the point of view introduced by Kregar generalized by one of us, based on the virial as potential energy and the splitting of the two-body potential energy operators into the sum of effective one-body operators (see below Equation 12) [12][13]. Briefly, in the zero approximation, disregarding for a moment the exchange and sub-shell corrections, as can be viewed in Equations 5 and 6, the average Coulomb energy of electron pairs is (the $i-t h$ electron is equally or more strongly bound that the $j-t h$ one)

$$
\left\langle i j\left|\frac{1}{r_{i j}}\right| i j\right\rangle=F^{0}(i j)=\left\langle i\left|\frac{g_{i j}}{r_{i}}\right| i\right\rangle+\left\langle j\left|\frac{f_{j i}}{r_{j}}\right| j\right\rangle
$$

so that the operator $1 / r_{i j}$ can be written as

$$
1 / r_{i j}=g_{i j} / r_{i}+f_{j i} / r_{j}
$$

where $g_{i j}$ and $f_{j i}$ are the external and internal screening parameters, respectively. When $i=j, f_{i i}=g_{i i} \equiv k_{i i}$. For a given value of $r$, inner screening is associated with electrons at $r^{\prime} \leq r$, outer screening is due to a shell of charge located at $r^{\prime}>r$, adding a constant to the potential $V(r)$. As it was shown in the References [12] and [13], the two parameters can be expressed in simple form in terms of $y=Z_{i} n_{j} / Z_{j} n_{i}$; $Z_{i}$ and $Z_{j}$ are the screened charge seen by each electron. A neat demonstration of this result can be found in reference [13].

To add the exchange and sub-shell corrections implies the changes

$$
g_{i j} \rightarrow g_{i j}\left(1-\varepsilon_{i j}\right), \quad f_{j i} \rightarrow f_{j i}\left(1-\varepsilon_{i j}\right)
$$


and can be accounted for as the ratio between the second term of the r.h.s. of Equations 5 and 6 and $F^{0}$.

Then, taking into account the exchange interaction and subshell effects, effective charges are given by

$$
Z_{i}=Z-\left[\sum_{j<i} q_{i} q_{j} f_{j i}+\sum_{j>i} q_{i} q_{j} g_{i j}+\left(q_{i}-1\right) k_{i i}\right]
$$

where $q_{i}, q_{j}$ are the occupation number of the respective subshells. As (see details in [13]), in terms of $y=$ $Z_{i} n_{j} / Z_{j} n_{i}$

$$
\begin{gathered}
g_{i j}=\left[\frac{1}{1+y}\right]^{3+2 l_{j}} \sum_{k} a_{k}\left[\frac{y}{1+y}\right]^{k}, \\
f_{j i}=\left[\frac{y}{1+y}\right]^{3+2 l_{i}} \sum_{k} b_{k}\left[\frac{1}{1+y}\right]^{k},
\end{gathered}
$$

a short and simple iterative procedure must be used for their determination. The iteration can be reduced to one or two steps if, as zero approximation, Slater-like rules are used as starting point for $Z_{i}$ (see below, section 3). Explicit expressions for the coefficients $a_{k}$ and $b_{k}$ can be found in reference [13].

Both the Layzer and the Kregar formulations ensures exact energy levels for $\mathrm{H}$-like ions in the non-relativistic regime; for He-like ions, $\sigma_{0}=5 / 16$, as deduced for the variational principle.

\subsection{Relativistic corrections}

From the Breit-Pauli Hamiltonian the relativistic shift operator $H_{R S}$ has five terms [4]:

$$
H_{R S}=H_{M C}+H_{D 1}+H_{D 2}+H_{O O}+H_{S S C}
$$

that signifies respectively: mass correction, one- and twobody Darwin, spin-spin contact and orbit-orbit terms. Far, the more important are the two first ones, that scale as $Z^{4}$.
These energy shifts can be estimated very simple and correctly as the sum of contributions

$$
E_{r e l}=-\frac{\alpha^{2}}{2} \sum_{i} \frac{Z^{4}}{n_{i}^{3}}\left(\frac{1}{l_{i}+0.5}-\frac{0.75}{n_{i}}-\delta\left(l_{i}, 0\right)\right) .
$$

The remaining terms scale as $Z^{3}$ and will be ignored in this work. An analysis is presented in the work by Safronova et al [2].

\section{Results and comparison with exper- iments and other calculations}

The comparison with experiment, in order to see the $Z$-dependence of experimental binding energies it is difficult, because there are relativistic effects dependent of $Z^{4}$ and $Z^{3}$ [8] and higher powers of $Z$. Therefore, we compare in first place our expansion with non-relativistic calculations. We verified that the non-relativistic values from the Cowan's code follow a dependence of the type

$$
E(N, Z)=Z^{2} E_{0}+Z E_{1}-E_{2}(1+a / Z) ;
$$

moreover, the Cowan values with relativistic corrections are very similar to the results from the Dirac-Hartree-Fock values from Huang et al. [15]

In Table 1, we show the center-of-gravity binding energies, including relativistic corrections for ground configurations, for atoms with closed (sub)shells. As we can see, our values are in very good agreement both with Hartree-Fock ([3], [15]) and experimental ones (up to $Z=20$, because there are not experimental results for all elements and ionization degrees).

In Fig. 1 we show the first ionization potential for the above cited elements, jointly with experimental values. The relation is of the order of two and better values can not be expected. In fact, the Layzer theory is valid, in principle, for large $Z$. Therefore this, the most rigorous test for our choice of the screening evaluation, is very promissory.

TABLE 1: Center of gravity binding energies (in $H t$ ) including relativistic corrections for ground configurations.

$\begin{array}{cccccc}\text { Element } & \text { This Work } & \text { Theory ([15]) } & \text { Experiment } & \frac{\text { This work }}{\text { Theory }} & \frac{\text { This work }}{\text { Experiment }} \\ \mathrm{He} & 2,85 & 2,86 & 2,90 & 0,99 & 0,99 \\ \mathrm{Be} & 14,59 & 14,57 & 14,67 & 1,00 & 0,99 \\ \mathrm{Ne} & 128,44 & 128,65 & 129,05 & 0,99 & 1,00 \\ \mathrm{Mg} & 200,06 & 199,89 & 200,31 & 1,00 & 1,00 \\ \mathrm{Ar} & 528,78 & 528,53 & 529,12 & 1,00 & 1,00 \\ \mathrm{Ca} & 680,24 & 679,51 & 680.14 & 1,00 & 1,00 \\ \mathrm{Zn} & 1793,04 & 1793,84 & N A^{*} & 0,99 & - \\ \mathrm{Kr} & 2787,80 & 2787,50 & N A & 1,00 & - \\ \mathrm{Sr} & 3177,06 & 3176,46 & N A & 1,00 & - \\ \mathrm{Cd} & 5580,65 & 5589,78 & N A & 0,99 & - \\ \mathrm{Xe} & 7423,77 & 7441,68 & N A & 0,99 & -\end{array}$




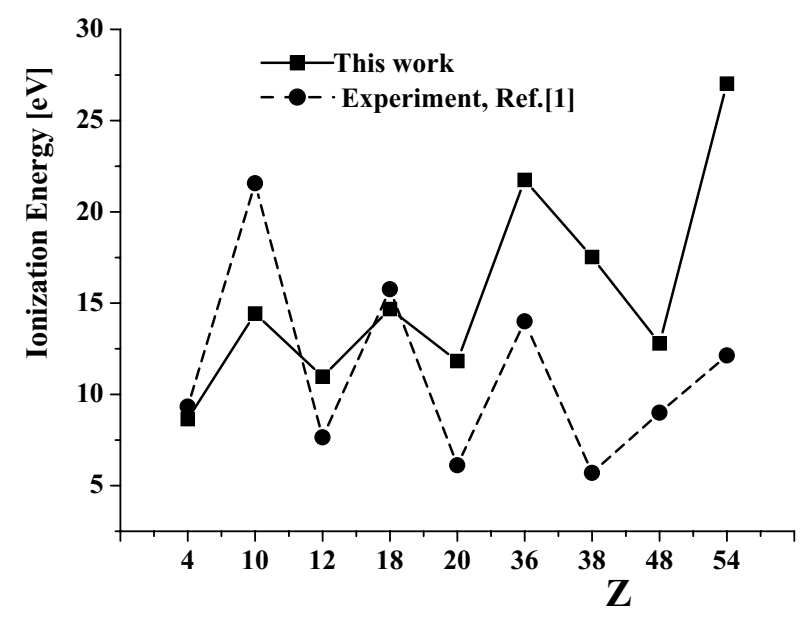

Figure 1. First ionization potentials for the elements with closed (sub)shells with $\mathrm{Z}$ between 1 and 54 .

TABLE 2: Ionization energies (in $e V$ ) for Ar isonuclear sequence.

$\begin{array}{cccc}\text { Z-N+1 } & \text { This work } & \text { Experimental [3] } & \frac{\text { This work }}{\text { Experimental }} \\ 1 & 14,72 & 15,76 & 0,93 \\ 2 & 27,34 & 27,63 & 0,99 \\ 3 & 41,20 & 40,74 & 1,01 \\ 4 & 56,22 & 59,81 & 0,94 \\ 5 & 72,34 & 75,02 & 0,96 \\ 6 & 89,55 & 91,01 & 0,98 \\ 7 & 127,75 & 124,32 & 1,03 \\ 8 & 147,15 & 143,46 & 1,03 \\ 9 & 418,49 & 422,45 & 0,99 \\ 10 & 480,70 & 478,69 & 1,01 \\ 11 & 545,47 & 538,96 & 1,01 \\ 12 & 612,81 & 618,26 & 0,99 \\ 13 & 682,70 & 686,11 & 0,99 \\ 14 & 755,17 & 755,75 & 0,99 \\ 15 & 850,34 & 854,78 & 0,99 \\ 16 & 918,27 & 918,00 & 1,00 \\ 17 & 4121,25 & 4120,00 & 1,00 \\ 18 & 4427,30 & 4426,00 & 1,00\end{array}$

In Table 2 are shown the ionization energies for the $A r$ isonuclear sequence; the relative values are near one.

In Table 3, are shown the ionization energies of the $\mathrm{Ar}$ isoelectronic sequence; as it is expected, the relation between our values and the Cowan values are in excellent agreement.

TABLE 3: Ionization potentials (in $e V$ ) for the isoelectronic sequence of Ar I.

$\begin{array}{lccc}Z & \text { This work } & \text { Experimental[3] } & \frac{\text { This work }}{\text { Experimental }} \\ 18 & 14,73 & 15,76 & 0,93 \\ 19 & 30,74 & 31,62 & 0,92 \\ 20 & 50,16 & 50,91 & 0,99 \\ 21 & 72,86 & 73,47 & 0,99 \\ 24 & 159,93 & 161,10 & 0,99 \\ 25 & 195,16 & 196,46 & 0,99 \\ 26 & 233,52 & 235,04 & 0,99 \\ 28 & 319,48 & 321,00 & 1,00 \\ 30 & 417,76 & 419,70 & 1,00 \\ 32 & 528,36 & 529,28 & 1,00\end{array}$

In Fig. 2 shows the binding energies of the internal subshells for $X e$; our values are compared with those from the theory and the experiment [15]; as can be seen, the agreement is very good.

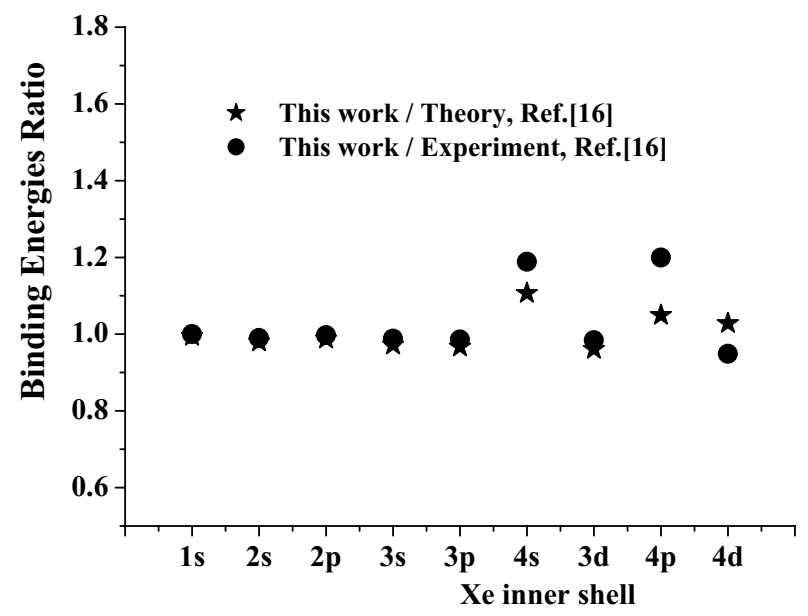

Figure 2. Binding energies ratio for the internal subshells of Xe.

As other example of complex transitions, we show in Table 4 transitions of the type $\Phi_{0} \Phi_{1}-2 p^{-1} 3 d \Phi_{0} \Phi_{1}$ as defined in Reference [2]: $\Phi_{0}=1 s^{2} 2 s^{2} 2 p^{6}, \Phi_{1}=3 d^{n} \Phi_{0}$. Both values are within $1 \%$, but it must be mentioned that the approach of Safronova et al. produces very bad values (negatives!) of the ionization energies for neutral and almost-neutral elements.

TABLE 4: Transitions of the type $\Phi_{0} \Phi_{1}-2 p^{-1} 3 d \Phi_{0} \Phi_{1}$ for the $\mathrm{Xe}$ atom compared with the values of reference[2].

$\begin{array}{cccc}\mathrm{N}^{*} & \text { This work } & {[2]} & \frac{\text { This work }}{[2]} \\ 10 & 4607,80 & 4647 & 0,992 \\ 11 & 4583,12 & 4621 & 0,992 \\ 12 & 4559,12 & 4595 & 0,992 \\ 13 & 4535,80 & 4568 & 0,993 \\ 14 & 4513,20 & 4542 & 0,994 \\ 15 & 4491,31 & 4516 & 0,995 \\ 16 & 4470,13 & 4490 & 0.996 \\ 17 & 4449,67 & 4464 & 0,997 \\ 18 & 4429,96 & 4438 & 0,998 \\ 19 & 4411,00 & 4412 & 1,000\end{array}$

${ }^{*} N=10+n$, being $n$ the number of $3 d$ electrons. 
TABLE 5. Scaled Slater integral $G^{1}(4 s 4 p)$ for the $\mathrm{Zn}$ isoelectronic sequence, in $\mathrm{cm}^{-1}$.

$\begin{array}{ccccc}Z & \text { Element } & G^{1}(4 s 4 p) \text { our } & G^{1}(4 s 4 p)_{H F R}[3] & \text { Ratio } \\ 35 & \text { Br 5+ } & 69636 & 86463 & 0.80 \\ 40 & \text { Zr 10+ } & 101611 & 121566 & 0.83 \\ 45 & \text { Rh } 15+ & 131118 & 153698 & 0.85 \\ 50 & \text { Sn 20+ } & 159552 & 184778 & 0.86 \\ 55 & \text { Cs } 25+ & 187504 & 215526 & 0.87 \\ 60 & \text { Nd 30+ } & 215079 & 246241 & 0.87 \\ 65 & \text { Tb 35+ } & 242547 & 277162 & 0.87 \\ 70 & \text { Yb 40+ } & 269801 & 308436 & 0.87 \\ 74 & \text { W 44+ } & 291636 & 333775 & 0.87\end{array}$

TABLE 6. Iteratively determined screening parameters $f_{j i}$ (values above diagonal) and $g_{i j}$ (values below diagonal) for electron pairs for $K r$-like ions, with $Z \rightarrow \infty$. Diagonal elements (bold characters) are $k_{i i}$.

$\begin{array}{ccccccccc} & 1 s & 2 s & 2 p & 3 s & 3 p & 3 d & 4 s & 4 p \\ 1 s & \mathbf{0 . 3 1 2 5} & 0.6924 & 0.8776 & 0.8095 & 0.9299 & 0.9967 & 0.8636 & 0.9539 \\ 2 s & 0.0258 & \mathbf{0 . 3 0 0 8} & 0.2230 & 0.6055 & 0.6466 & 0.7756 & 0.7290 & 0.7630 \\ 2 p & 0.0149 & 0.3668 & \mathbf{0 . 3 4 9 2} & 0.6617 & 0.6821 & 0.8452 & 0.7650 & 0.7887 \\ 3 s & 0.0068 & 0.0528 & 0.0471 & \mathbf{0 . 2 9 8 8} & 0.2435 & 0.2164 & 0.5512 & 0.5737 \\ 3 p & 0.0034 & 0.0674 & 0.0635 & 0.3121 & \mathbf{0 . 3 1 0 4} & 0.2561 & 0.5861 & 0.5914 \\ 3 d & 0.0002 & 0.0562 & 0.0368 & 0.4213 & 0.4066 & \mathbf{0 . 3 7 6 5} & 0.6281 & 0.6391 \\ 4 s & 0.0026 & 0.0189 & 0.0167 & 0.0776 & 0.0746 & 0.0678 & \mathbf{0 . 2 9 8 2} & 0.2545 \\ 4 p & 0.0011 & 0.0238 & 0.0223 & 0.0900 & 0.0855 & 0.0784 & 0.2889 & \mathbf{0 . 2 9 8 7}\end{array}$

\subsection{Calculation of terms and energy levels}

The results shown up to this point allow the calculation of the configurations average energies [3]. This may be enough for calculating, for example, the array oscillator strength, which is frequently used for statistical treatments of dielectronic recombination and collisional excitation, in plasma physics and astrophysical applications. For the calculation of the multiplet oscillator strength, the terms are needed and, consequently, the Slater integrals, $F^{k}(i j)$ and $G^{k}(i j)$, which scale with $Z_{e f f}$. Thus, $F^{k}(i j ; Z)=\left(Z-\sigma_{n l}\right) \times$ $F^{k}(i j ; Z=1), G^{k}(i j ; Z)=\left(Z-\sigma_{n l}\right) \times G^{k}(i j ; Z=1)$. Values of these integrals for $Z=1$ can be found in the book by Condon-Odabasi [14]. In particular, and as an example, $G^{1}(4 s 4 p ; Z=1)=0.02444565 R y$.

Table 5 shows the values of $G^{1}(4 s 4 p ; Z)=$ $0.02444565\left(Z-\sigma_{n l}\right)$ for the configuration $4 s 4 p$ of $Z n-$ like with $Z=30-74$. These results are compared with the relativistic Hartree-Fock approximation (HXR). It is very important take into account that in practice, in order to give a better accordance with experimental spectroscopy, the theoretical Slater integrals must be multiplied by "fudge" factors lesser than 1 [3].

\subsection{Slater-like rules and constant values for the $g_{i j}$ 's and the $f_{j i}$ 's for $Z \rightarrow \infty$.}

As stated above (see equation 11), a desirable property for $\sigma$ is that for $Z \rightarrow \infty, \sigma \rightarrow \sigma_{0}$. This is verified by our approach and implies that for $Z \rightarrow \infty$, the values of $f_{i j}$ and $g_{i j}$ trend themselves to constant values, which we can denominate $f_{i j}^{\infty}$ and $g_{i j}^{\infty}$. An example is given in Table 6 for the particular case of the orbitals of $K r$-like ions. These values, once known, can be stored and reliably reused either in calculations for high ionization stages or, as input parameter to reduce the number of iterations needed to achieve convergency in Eq. 14. For example, using Eq. 13, $\sigma_{1 s}=0.4973$, $\sigma_{2 s}=5.0253$, etc.

\section{Conclusions}

In this work we showed that the Layzer expansion is not only a theoretical framework but also a very convenient quantitative method when a correct approach for screening parameter calculations is given. We select the point of view introduced by Kregar, generalized by one of us, taking into account subshell and exchange corrections. With our approach, we always obtain positive ionization potentials, even for neutral atoms, the most difficult test. This fact is easily understood because the Layzer theory is an expansion valid for large $Z$. In practice, for slightly ionized atoms (as low as three times), results are very accurate accordingly with the main scope of this work: plasma modelling and collision experiments. It it important to point out that in the present work no fitting procedure is used although, if we want to extend the method for neutral and almost neutral atoms, a correction factor (very close to 1 ) for the $\sigma^{\prime} s$ could be used. Moreover, it should be mentioned that, although a set of $f_{j i}$ and $g_{i j}$ constants could be used (which we have called $f_{i j}^{\infty}$ and $g_{i j}^{\infty}$ ), better results are obtained if the $f_{j i}$ and $g_{i j}$ are handled as parameters. Our approach assure this property taking into account the variation with $Z$ and $N$. However, it is true that for sufficiently ionized atoms, the use of constants provides a very good approximation, useful for plasma modeling and ion-surface collision experiments [2]. 


\section{References}

[1] J.G. Rubiano, R. Rodríguez, J.M. Gil, F. H. Ruano, P. Maretel, and E. Mínguez, J. Quant. Spectrosc. Radiat. Transfer, 72, 575 (2002)

[2] U. I. Safronova et al., Phys. Scripta 47, 364 (1993); M.W. Clark et al, Phys. Rev. A 47, 3983 (1993).

[3] R. D. Cowan, The Theory of Atomic Structure and Spectra (University of California Press, Berkeley and Los Angeles, 1981).

[4] C. Froese Fischer, T. Brage, and P. Jonsson, Computational Atomic Structure (IoP Publishing, Bristol, 1997).

[5] D. Layzer, Ann. Phys. 8, 271 (1959); D. Layzer et al., Ann. Phys. 29, 101 (1964).

[6] H.O. Di Rocco, Il Nuovo Cimento D 20, 131 (1998).

[7] G. Faussurier, C. Blancard, and A. Decoster, J. Quant. Spectrosc. Radiat. Transfer 58, 233 (1997).
[8] D. Layzer, Int. J. Quantum Chemistry 1 S, 45 (1967).

[9] R. M. More, J. Quant. Spectrosc. Radiat. Transfer 27, 345 (1982).

[10] F. Perrot, Phys. Scripta 39, 332 (1989).

[11] P. Martel, J.G. Rubiano, J.M. Gil, L. Doreste, and E. Mínguez, J. Quant. Spectrosc. Radiat. Transfer, 60, 623 (1998).

[12] M. Kregar, Phys. Scripta 29, 438 (1984), Phys. Scripta 31, 246 (1985).

[13] H. O. Di Rocco, Braz. J. Phys. 22, 227 (1992).

[14] E. U. Condon and H. Odabasi, Atomic Structure (University Press, Cambridge, 1980).

[15] K.-N. Huang et al., At. Data Nucl. Data Tables 18, 249 (1976).

[16] H. O. Di Rocco, Revista Mexicana de Física 48, 76 (2002). 